\section{Large computers key to aggregation phenomena}

SIR-We thank you for devoting a part of a News and Views editorial' to one of our recent papers ${ }^{2}$. Our paper describes a reversible process of particle-cluster aggregation-disaggregation in which particles at the periphery of a cluster may escape and come back again after undergoing a random walk in space. We agree with your argument that this does not truly represent aggregation at equilibrium, but the motivation behind our work was somewhat different. We aimed to describe more exactly some kind of dynamic equilibrium or steady state in which a flux of incoming diffusing particles strictly compensates a flux of outgoing diffusing particles.

In this spirit, our work was and attempt to include restructuring processes in the existing models of aggregation (other attempts are by Meakin and Jullien ${ }^{3}$ as you quoted, but also P. Meakin ${ }^{4}$ ). This was a first step before studying cluster-cluster aggregation-disaggregation processes, where bonds can be annealed randomly all along the diffusion-sticking mechanism. This last model was recently studied by Kolb 5 .

We agree that our main result (our clusters have apparently the same fractal dimension as animals) is quite intricate. When describing our model to several colleagues, we got a large range of guesses and speculations; they predicted either DLA, or Eden, or animals and so on. This is why our paper included a detailed discussion explaining that it was none of those. However, since we found a fractal dimension close to the one for animals, we insisted on the comparison with this at-equilibrium model.

Interestingly, however, cluster-cluster aggregation-disaggregation, as demonstrated by Kolb, also yields the same fractal dimension. Moreover, in the past ${ }^{6}$, Stauffer used a process very similar to ours (but without any diffusion) in which particles are randomly released from the perimeter and then put back randomly on it (this is a "chemical" version of our aggregationdisaggregation model). Stauffer claimed that his objects were in the same universality class as animals.

It is very surprising that these three aggregation-disaggregation models all share the same fractal dimension, since different rules might be expected to induce different statistics. The cluster statistics for small clusters are clearly not the same as for large clusters, so why do they peak to an average configuration in the thermodynamic limit of very large clusters?

To conclude, we think that as long as there is no analytical progress in these fields, large size computer simulations will be the only tool enabling us to understand and classify the models. Moreover, they can define the basis for a search for analytical solutions. In the case of aggregation phenomena the two basic models (particle-cluster and cluster-cluster aggregation) are now well characterized and they must be modified and extended to better apply to experiments (compare for example the recent attempt to take into account cluster polarizability ${ }^{7}$, to explain the experiment by Hurd and Schaefer $\left.{ }^{8}\right)$. It is however essential that in the course of these improvements, the models do not lose their high degree of simplicity and generality.

R. BOTET

Université de Paris-Sud,

R. JULLIEN

Centre d'Orsay,

Laboratoire de Physique des Solides,

91405 Orsay, Paris, France

1. Maddox, J. Nature 318, 229 (1985).

2. Botet, R. \& Jullien, R. Phys. Rev. Lett. 55, 1943-1946 (1985).

. Meakin, P. \& Jullien, R. J. Physique 46, 1543-1552 (1985).

. Meakin, P. J. chem. Phys. 83, 3645-3649 (1985)

Kolb, M. J. Phys. A, (in the Press).

. Stauffer, D. Phys. Rep. 54, 1-74 (1979)

. Jullien, R. Phys. Rev. Lett. 55, 1692-1697 (1985).

. Hurd, A. \& Schaefer, D. Phys. Rev. Lett. 54, 1043-1046 (1985).

\section{Minimum energy configurations}

SIR-Berezin ${ }^{1}$ has recently pointed out that the minimum energy configuration for a system of $m$ identical point charges interacting via the Coulomb potential $1 / r$ and confined to a circular region in a plane has all the charges on the circumference only if $m \leqslant 11$. For $m>11$ some of the charges are in the interior. (Specifically, Berezin showed that the energy of configuration $\mathrm{B}(m-1$ charges uniformly distributed around the circumference plus one charge at the centre) is lower than that of configuration A ( $m$ charges uniformly distributed around the circumference) for $m>11$. We have extended Berezin's work and have shown that at $m=17$ it is advantageous to have two particles in the interior. Note also that for $m \rightarrow \infty$ the problem becomes that of the conducting disk.) $\mathrm{He}$ conjectured that a similar result might obtain in three dimensions. Unfortunately one can show, using the fact that the Coulomb potential is harmonic in three dimensions $^{2}$, that the minimum energy configuration for a system of $m$ identical point charges confined to a region of space has all the charges on the surface of the region, independent of the number of particles $m$. If, however, we change Berezin's conjecture slightly, the problem again becomes interesting.

We ask: what is the minimum energy configuration for a system of $m$ identical particles interacting via a non-harmonic potential $1 / r^{n}, n>1$, and confined to a spherical volume? (This is why Berezin's two dimensional system has charge in the interior - the potential $1 / r$ is not harmonic in two dimensions. If, as MacGowan ${ }^{3}$ suggests, one uses the potential $\ln (r)$, harmonic in two dimensions, the charge all lies on the circumference.) Clearly, for $m=2,3,4$, the particles will lie on the surface of the sphere independent of $n$. For higher $m$ the problem becomes more difficult.

Melnyk et al.${ }^{4}$ have studied in detail the problem of the minimum energy configuration for systems of particles constrained to the surface of a sphere. Calculations based on their configurations lead us to conjecture that the minimum energy configuration has all the particles on the surface for $m \leqslant 12$, independent of the power $n$ of the potential. For $m=13$ and higher, however, the situation is different. This can be seen most easily in the short range limit $n \rightarrow \infty$. In this limit the energy is dominated by nearest neighbour interactions. At $m=13$ the nearest neighbour distance becomes less than 1 (in units of the sphere radius) and it becomes advantageous to remove one particle from the surface and place it at the centre of the sphere (leading to a minimum energy configuration of icosahedron plus one at the centre). We have shown that this situation persists if the power of the interaction $n$ is as small as approximately 6.3 .

For $n<6.3$ the minimum energy configuration has all the particles on the surface in the configuration described by Melnyk et al. ${ }^{4}$. It seems likely that a similar situation will persist for still larger numbers of particles: for low powers of the interaction all the particles will be on the surface; for higher powers some particles will be in the interior. A complete mapping of the " $m-n$ phase plane", will, however, involve much detailed study.

M.G. CALKIN

D. KIANG

Department of Physics,

D.A. Tindall

Dalhousie University,

Halifax, Nova Scotia,

Canada B3H $3 J 5$

1. Berezin, A.A. Nature 315, 104 (1985)

2. Kellog, O.D. Foundations of Potential Theory 223 (Springer, Berlin, 1929).

MacGowen, D. Nature 315, 635 (1985)

4. Melnyk, T.W.. Knop, O \& Smith, W.R. Can. J. Chem. 55, 1745-1761 (1977)

\section{Scientific Correspondence}

Scientific Correspondence is intended to provide a forum in which readers may raise points of a rather technical character which are not provoked by articles or letters previously published (where the Matters Arising section remains appropriate). 